\title{
Zr-metal adhesion on graphenic nanostructures
}

\author{
Y. Sanchez-Paisal, ${ }^{1}$ D. Sanchez-Portal, ${ }^{1}$ N. Garmendia, ${ }^{2}$ R. Muñoz, ${ }^{2}$ I. Obieta, ${ }^{2}$ J. Arbiol, ${ }^{3}$ \\ L. Calvo-Barrio, ${ }^{3}$ and A. Ayuela ${ }^{1, a)}$ \\ ${ }^{1}$ Donostia International Physics Center (DIPC) and Unidad Física de Materiales, \\ Centro Mixto CSIC-UPV/EHU, 20080 Donostia-San Sebastián, Spain \\ ${ }^{2}$ INASMET-Tecnalia, Mikeletegi Pasealekua 2, E-20009 Donostia-San Sebastián, Spain \\ ${ }^{3}$ TEM-MAT, Serveis Cientificotècnis, Universitat de Barcelona, 08028 Barcelona, Spain
}

(Received 20 February 2008; accepted 10 July 2008; published online 4 August 2008)

\begin{abstract}
Our high resolution transmission electronic microscopy studies of multiwall carbon nanotubes show, after the growth of zirconia nanoparticles by a hydrothermal route, the presence of surface $\mathrm{Zr}$, forming an atomically thin layer. Using first-principles calculations we investigate the nature of the $\mathrm{Zr}-\mathrm{C}$ interaction, which is neither ionic nor covalent, and the optimal coverage for the $\mathrm{Zr}$ metal in a graphene flake. This preferred coverage is in agreement with that deduced from electron energy loss spectra experiments. We show also that the amount of charge transferred to the $\mathrm{C}$ layer saturates as the $\mathrm{Zr}$ coverage increases and the $\mathrm{Zr}-\mathrm{C}$ bond becomes weaker. (C) 2008 American Institute of Physics. [DOI: 10.1063/1.2966373]
\end{abstract}

Carbon nanotubes (CNTs) are among the most important constituent materials in nanotechnology applications. ${ }^{1}$ As a result of technological advances in the production and processing of CNT, they are widely used to produce reinforced composite materials, such as $\mathrm{ZrO}_{2} / \mathrm{CNT}$ compounds. ${ }^{2,3}$ Notwithstanding remarkable success in fabricating these devices, the atomic processes that govern the adhesion of dopants and their interaction with the native graphene structure are not fully understood. ${ }^{4}$ This is particularly true for the adsorption of Zr, an early transition metal, on CNTs or graphene sheets. It is one of the focal points of this letter to study the $\mathrm{Zr}-\mathrm{C}$ interaction.

Whereas several groups are interested in the zirconia/ CNT composites, the nature of the $\mathrm{ZrO}_{2}-\mathrm{CNT}$ interaction has not been clarified. In particular, the existence of $\mathrm{Zr}-\mathrm{C}$ bond has not been demonstrated to date. Using zirconium as catalyst during nanotube growth leads to nanostructures with zirconia crystals of about $15 \AA$ in size anchored in the outer wall. ${ }^{5-7}$ However, our experiments show that this picture is incomplete and the true situation is more complex and intriguing. The experiments described below show that the $\mathrm{Zr}-\mathrm{C}$ interaction can play a key role in the growth and anchoring of these particles.

We first show the existence of a $\mathrm{Zr}$ layer in the outer part of the CNT walls. When depositing $\mathrm{Ti}$ (or $\mathrm{Al}$ ) on graphite materials, it is known that the formation of carbides is mainly due to the $\{100\}$ and $\{110\}$ reactive planes. However, the hexagonal planes are chemically stable compared to the reactive planes at the experimental temperatures studied here. Moreover, in nanotubes, the graphitic basal plane is stable against depositing $\mathrm{Ti}$ (even $\mathrm{Al}$ ) at higher temperatures and under pressure. ${ }^{8}$ Our experimental studies use samples obtained via hydrothermal crystallization of the zirconium hydroxide $\mathrm{Zr}(\mathrm{OH})_{4}$ in the presence of the multiwall nanotubes (MWNTs). ${ }^{9}$ First, we dissolve the precursor $\mathrm{Zr}(\mathrm{OH})_{4}$ in distilled water by stirring and the $p \mathrm{H}$ is modified to obtain a basic dissolution. Then, we add the nanotubes and ultra-

\footnotetext{
${ }^{\text {a) }}$ Author to whom correspondence should be addressed. Electronic mail: swxayfea@sw.ehu.es.
}

sonicate the mixture. The $\mathrm{Zr}(\mathrm{OH})_{4}$ aqueous solution with MWCNT thereby obtained is sealed in a stainless steel Teflon autoclave and is kept at $240{ }^{\circ} \mathrm{C}$ for $6 \mathrm{~h}$ to carry out the hydrothermal reaction. Finally, we dried the solution in a stove at $70{ }^{\circ} \mathrm{C}$ for one day and the final product is characterized. This procedure ensures that the $\mathrm{Zr}$ additions would remain on the external part of the CNT walls.

We have performed a detailed structural and morphological characterization of the samples by transmission electron microscopy. The different structures [Fig. 1(a)] are identified with high resolution transmission electron microscope (HRTEM) micrographs. To obtain the HRTEM results, a field emission gun microscope JEOL 2010F was used which works at $200 \mathrm{kV}$ and has a point resolution of $0.19 \mathrm{~nm}$. Electron energy loss spectra (EELS), obtained with a Gatan Image Filter (GIF 2000) coupled to the microscope, have an energy resolution of $1.2 \mathrm{eV}$. In order to improve the chemical analysis, we carried out high angular annular dark field scanning transmission electron microscopy (STEM) on our samples. In the HRTEM image of the $\mathrm{ZrO}_{2}$ /CNT composite, $\mathrm{Zr}$ carbide formation by the chemical reaction between $\mathrm{Zr}$ and CNT is not observed.

The HRTEM images taken in the nanoparticles attached to the CNT show the characteristic features of the $\mathrm{ZrO}_{2}$ spectrum, proving that they are indeed zirconia particles. However, something interesting is observed in the areas apparently not covered. In Fig. 1(b), we display the EELS along a section of the wide MWNTs. The spectra show a small peak corresponding to $\mathrm{Zr}$ on the walls, without the presence of oxygen or iron (see caption). A deconvolution of the spectra along the line is given in panel (c). To obtain the $\mathrm{Zr} / \mathrm{C}$ (surface) ratio, we multiply by the averaged number of carbon atoms of the MWNT section (assuming ten layers). The proportion of $\mathrm{Zr}$ (ranging from 10\% to 20\%) is low compared with surface $\mathrm{C}$, and it is clearly larger than the average number of vacancies on the surface of MWNTs. There is always an appreciable concentration of $\mathrm{Zr}$ on the $\mathrm{C}$ plane, but the $\mathrm{Zr} / \mathrm{C}$ (surface) ratio is clearly lower than $50 \%$. The interplay among $\mathrm{Zr}-\mathrm{Zr}, \mathrm{Zr}-\mathrm{C}$, and $\mathrm{C}-\mathrm{C}$ interactions explains this limit. We shall return to this below. 


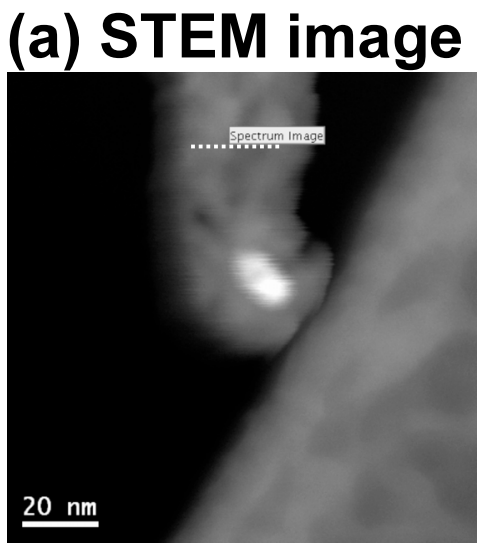

(b) EELS spectrum

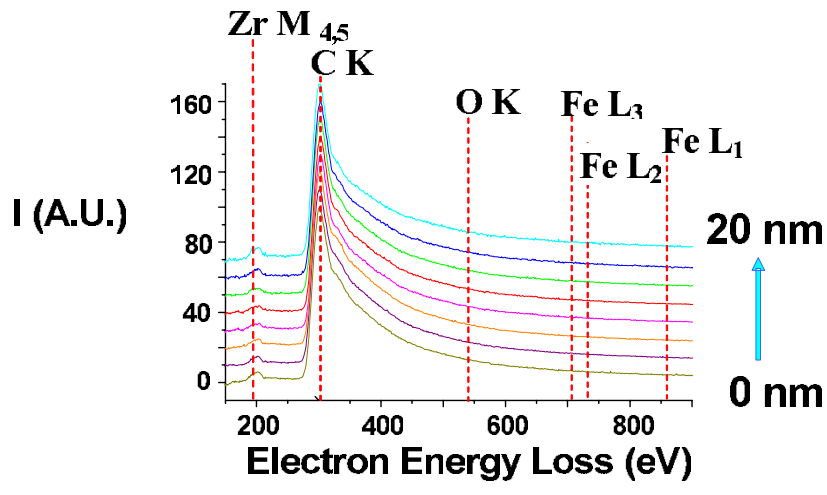

(c) \% Zr along dashed line

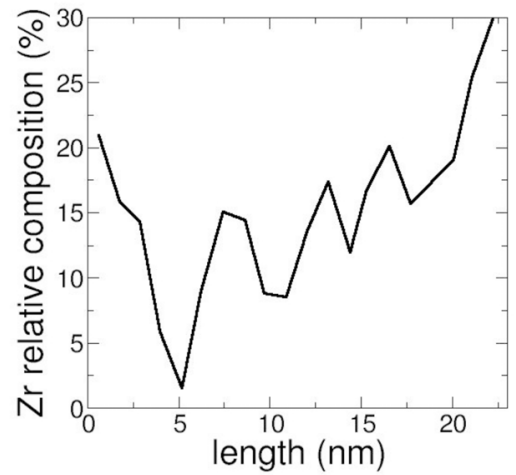

FIG. 1. (Color online) (a) STEM image of CNT in a region free of zirconia particles. The bright spot enclosed by the CNT is a catalyst particle composed of iron. The top finger structure are MWNTs with 10-20 layers. Across the dashed line we obtain the EELS on transmission shown in part (b). The beam is $0.2 \mathrm{~nm}$ wide and a spectrum is measured every $2.5 \mathrm{~nm}$. The vertical dashed-red lines show transitions corresponding to the elements that are expected to be present in the sample. Neither iron or oxygen are present. Notice, however, the large $\mathrm{Zr}$ content. (c) $\mathrm{Zr} / \mathrm{C}$ (surface) distribution profile on the tube, along the same dashed line in panel (a).

We next turn to the $\mathrm{Zr}-\mathrm{C}$ adsorption. We have investigated a number of adsorption sites and coverage on graphene sheet-stable "graphitic basal plane"-which is a good approach for the surface of wide MWCNTs such as those found in our sample.

We have carried out spin-density-functional calculations using the SIESTA code. ${ }^{10}$ The generalized gradient approxi-

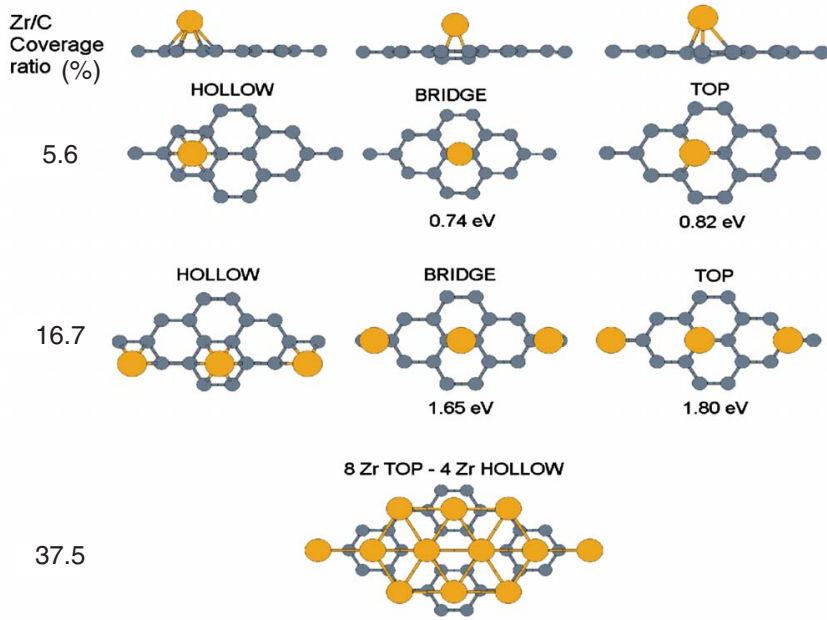

50.
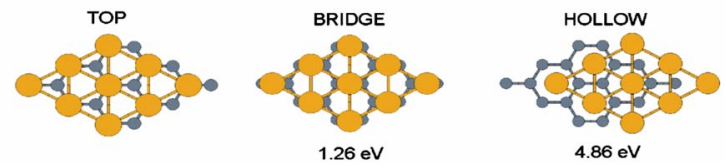

FIG. 2. (Color online) Geometries for different $\mathrm{Zr} / \mathrm{C}$ coverage ratios. Below each configuration, the total energy difference with respect the ground state for each coverage is given in eV. Carbon atoms are small-dark circles and zirconium ones are large light. The results for the $4 \times 4$ cell are not shown because the same total energy differences are obtained for $\mathrm{Zr} / \mathrm{C}=5.6 \%$.

mation for the exchange and correlation follows the PerdewBurke-Ernzerhof approach. ${ }^{11}$ The explicit treatment of electronic core states is avoided by using the pseudopotentials of Troullier-Martins. ${ }^{12}$ The basis set is double- $\zeta$ polarized and includes the $4 p$ semicore electrons of $\mathrm{Zr}$. The calculations are done using $3 \times 3$ and $4 \times 4$ graphene supercells containing 18 and 32 carbon atoms with different zirconium coverages as shown in Fig. 2, ranging between $5.6 \%$ and $50 \%$. The $\mathrm{Zr}$ atoms are arranged at hollow, bridge, and top positions, in clustered and homogeneous geometries. We used $40 k$-points in the irreducible Brillouin zone and a mesh cutoff of $180 \mathrm{Ry}$ that guarantees an energy convergence below $10 \mathrm{meV} /$ atom. We fully relax the atomic coordinates and cell vectors until the forces are lower than $0.04 \mathrm{eV} / \AA$. Then, the binding energy $E_{B}$ per $\mathrm{Zr}$ atom covering the $\mathrm{C}$ plane is calculated as

$$
\begin{aligned}
E_{B}= & -\left\{E_{T}[\mathrm{Zr}-\text { graphene }]-E_{T}[\text { graphene }]\right. \\
& \left.-N E_{T}[\mathrm{Zr}]\right\} / N,
\end{aligned}
$$

where $E_{T}[$ system $]$ is the total energy of the system and $N$ is the number of $\mathrm{Zr}$ atoms per cell.

We first investigate $\mathrm{Zr}$ on graphene for low coverage $\mathrm{Zr} / \mathrm{C}$ ratios of $5.6 \%$. We find that the hollow position for a $\mathrm{Zr}$ atom is more stable than top and bridge ones by only 0.74 and $0.82 \mathrm{eV}$, respectively. ${ }^{13}$ Thus, the barrier between hollow states is at least $0.74 \mathrm{eV}$, and there could be some diffusion at room temperature. The binding energy per $\mathrm{Zr}$ atom is $3.06 \mathrm{eV}$ in the hollow position.

For high coverages $(\mathrm{Zr} / \mathrm{C}=50 \%)$, we found the reverse order between the hollow and top positions. For high coverages, the binding energy of hollow $\mathrm{Zr}$ with $\mathrm{C}$ is $4.66 \mathrm{eV}$ and lower than the binding energy of top $\mathrm{Zr}$ which is $5.20 \mathrm{eV}$. Hence, if we compared with the $\mathrm{Zr}$ binding we find in the fully relaxed hexagonal $\mathrm{Zr}$ plane, $5.40 \mathrm{eV}$, we conclude that the binding energy of $\mathrm{Zr}$ is larger with nearest-neighbor zirconium atoms than with the graphene sheet. The $\mathrm{Zr}$ overlayer 


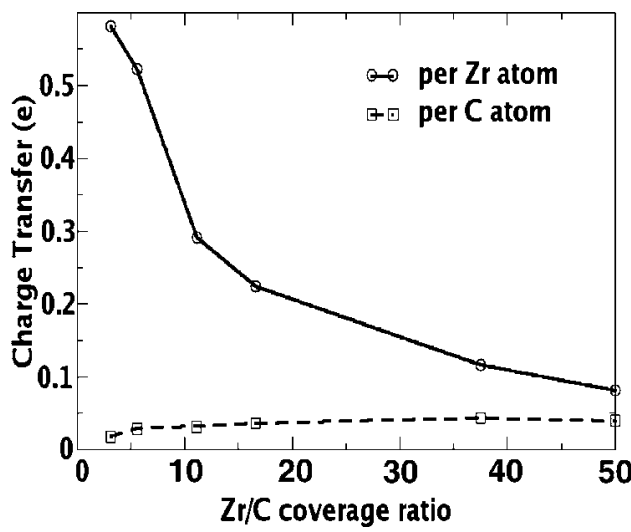

FIG. 3. Charge transfer vs $\mathrm{Zr} / \mathrm{C}$ coverage ratio. All $\mathrm{Zr} / \mathrm{C}$ coverage ratios are for hollow positions except the point with a $\mathrm{Zr} / \mathrm{C}$ coverage ratio of $37.5 \%$, which is a mixed hollow/top configuration.

is under compressive stress at such large coverages. We have also examined at higher $\mathrm{Zr} / \mathrm{C}$ ratios and find that the graphene layer breaks and forms carbides.

The height of the $\mathrm{Zr}$ atoms over the graphene plane strongly increases with the $\mathrm{Zr}$ coverage in the case of hollow positions, while remaining almost constant for top positions $(2.24 \AA$ for $\mathrm{Zr} / \mathrm{C}=5.6 \%$ and $2.38 \AA$ for $\mathrm{Zr} / \mathrm{C}=50 \%)$. In hollow positions the height of $\mathrm{Zr}$ atoms to graphene plane at low coverages contrasts with that at high coverages. For low coverages, $\mathrm{Zr}$ lies tightly bound to $\mathrm{C}$ with a $\mathrm{Zr}-\mathrm{C}$ height of $1.89 \AA$ A. In contrast, for high coverages, $\mathrm{Zr}$ lies weakly bound to $\mathrm{C}$ with a height of $2.61 \AA$.

We suggest a candidate for optimum coverage with mixed top and hollow positions ( 2 top/1 hollow) and a $\mathrm{Zr} / \mathrm{C}$ ratio of $37.5 \%$, as seen in Fig. 2. We find its binding energy per $\mathrm{Zr}$ to be $6.08 \mathrm{eV}$, which is more stable than previous cases by $0.68 \mathrm{eV}$. This coverage seems to be the optimal one and is slightly larger that the one found in the previous experimental data, in the range of $10 \%-20 \%$ but smaller than $50 \%$.

We next explain the bonding mechanism as a function of the coverage. It has been found for metals on graphitic nanostructures that the metals transfer charge to the hexagonal $\mathrm{C}$ cage. ${ }^{4,14}$ Using Mulliken population analysis we compute the charge in the atoms as shown in Fig. 3. We find that the $\mathrm{Zr}$ atoms lose charge to the $\mathrm{C}$ atoms and the charge transfer per $\mathrm{Zr}$ atom decreases as coverages increases, while the extra charge per $\mathrm{C}$ is almost constant. In other words, the graphene plane accepts a maximum of electrons when $\mathrm{Zr}$ coverage is increased. According to similar analysis for all configura- tions (top, hollow, and bridge) at each coverage, the differences in the values of charge transfers are around $10 \%$. Clearly, this means that our conclusions slightly depend on the configuration. Finally, we point out that in every case the charge transfer moves from $5 s$ orbitals of $\mathrm{Zr}$ to $2 p_{z}$ orbitals in graphene. We also find that this charge is delocalized in graphene.

In summary, we have found that $\mathrm{Zr}$ covers the wall of CNTs during the growth of $\mathrm{ZrO}_{2}$ nanoparticles using a hydrothermal route. Ab initio calculations give a preferred $\mathrm{Zr} / \mathrm{C}$ coverage ratio of $37.5 \%$ in good agreement with the EELS experiments. Here, the $\mathrm{Zr}$ atoms are both on top and hollow positions. This might prove important to understand the interaction of transition metals with CNTs and, in particular, the growth mechanism of $\mathrm{ZrO}_{2}$ nanoparticles for technological applications.

We want to acknowledge the support by the ETORTEK (NANOMAT) program of the Basque government, the Intramural Special Project (Reference No. 2006601242), the Spanish Ministerio de Ciencia y Tecnología (MCyT) of Spain (Grant No. Fis 2007-66711-C02-C01), and the European Network of Excellence NANOQUANTA (NM4-CT2004-500198). Y.S.P. gratefully acknowledges his DIPC grant.

${ }^{1}$ S. Ijima, Nature (London) 354, 56 (1991).

${ }^{2}$ G. L. Hwang and K. C. Hwang, J. Mater. Chem. 11, 1722 (2001).

${ }^{3}$ E. Flahaut, A. Peigney, Ch. Laurent, Ch. Marliere, F. Chastel, and A. Rousset, Acta Mater. 48, 3803 (2000).

${ }^{4}$ J. I. Martinez, M. J. López, and J. A. Alonso, J. Chem. Phys. 123, 074303 (2005).

${ }^{5}$ F. Lupo, R. Kamalakaran, C. Scheu, N. Grobert, and M. Rühle, Carbon 42, 1995 (2004).

${ }^{6}$ L. Huang, S. P. Lau, Y. B. Zhang, B. K. Tay, and Y. Q. Fu, Nanotechnology 15, 663 (2004).

${ }^{7}$ Y. Shan and L. Gao, Nanotechnology 16, 625 (2005).

${ }^{8}$ T. Kuzami, O. Ujie, H. Ichinose, and K. Ito, Adv. Eng. Mater. 2, 416 (2000).

${ }^{9}$ N. Garmendia, L. Bilbao, R. Muñoz, L. Goikoetxea, A. García, I. Bustero, B. Olalde, N. Garagorri, and I. Obieta, Key Eng. Mater. 361, 775 (2008).

${ }^{10}$ J. M. Soler, E. Artacho, J. D. Gale, A. Garcia, J. Junquera, P. Ordejon, and D. Sánchez-Portal, J. Phys.: Condens. Matter 14, 2745 (2002).

${ }^{11}$ J. P. Perdew, K. Burke, and M. Ernzerhof, Phys. Rev. Lett. 77, 3865 (1996).

${ }^{12}$ N. Troullier and J. L. Martins, Phys. Rev. B 43, 1993 (1991).

${ }^{13} \mathrm{We}$ are commenting only energies from the ground state energy and its energy differences with the first low lying configuration. These are robust and large enough so that the basis set superposition error would not change the trends. Other configurations are almost degenerate and we are not commenting on them.

${ }^{14}$ E. Durgun and S. Ciraci, Phys. Rev. B 74, 125404 (2006). 